
rural

\title{
Le lien des agriculteurs aux lieux et aux autres habitants. Une évolution majeure à observer
}

Farmers' relation to places and others rural inhabitants: a major social change to observe

\section{Nicole Mathieu}

\section{(2) OpenEdition} Journals

\section{Édition électronique}

URL : http://journals.openedition.org/economierurale/2280

DOI : $10.4000 /$ economierurale.2280

ISSN : 2105-2581

\section{Éditeur}

Société Française d'Économie Rurale (SFER)

\section{Édition imprimée}

Date de publication : 10 septembre 2007

Pagination : 129-133

ISSN : 0013-0559

Référence électronique

Nicole Mathieu, "Le lien des agriculteurs aux lieux et aux autres habitants. Une évolution majeure à observer », Économie rurale [En ligne], 300 | Juillet-août 2007, mis en ligne le 12 novembre 2009, consulté le 21 avril 2019. URL : http://journals.openedition.org/economierurale/2280 ; DOI : 10.4000/ economierurale. 2280 


\title{
Le lien des agriculteurs aux lieux et aux autres habitants Une évolution majeure à observer
}

\author{
Nicole MATHIEU \\ Directeur de recherche émérite au CNRS, Université Paris 1, UMR Ladyss \\ Ancien vice-président de la SFER, ancien membre du Comité de rédaction de la revue
}

Avant d'exposer les faits majeurs de société donnant lieu à quelques enseignements sur l'évolution du monde agricole et rural et imaginer l'avenir, Nicole Mathieu s'interroge sur ce qui est nécessaire au chercheur en sciences sociales : être à la fois un observateur et un analyste impliqué dans la vie rurale. C'est à ce titre seulement que le chercheur devient un témoin privilégié et reconnu pour expliquer l'évolution de l'économie et de la société rurale française. En passant de l'observation du local au global, le constat le plus important pour l'auteur est celui du changement dans le mode d'habiter les territoires ruraux. Mais, de ce constat émerge une autre interrogation : comment construire une nouvelle cohabitation du milieu rural ?

$\mathbf{L}$ 'idée est bonne de signifier la continuité symbolique d'Économie rurale en demandant pour le numéro 300 à des « observateurs, analystes, acteurs, témoins des évènements qui ont marqué et qui marquent l'évolution de l'agriculture » de s'exprimer sur « le fait historique ou l'évolution économique ou sociologique » qui les a le plus étonné et dont ils tirent le plus d'enseignement pour imaginer l'avenir de la société comme de leur métier de chercheur'.

Avant de me plier à l'exercice, il me faut d'abord dire qu'Économie rurale constitue pour moi une tribune scientifique privilégiée pour rendre compte du difficile métier d'observateur et d'analyste du «monde rural ». Le chercheur en sciences sociales a en effet besoin d'un lieu de publication où il puisse exprimer, dans sa complexité, la

\footnotetext{
1. Pour avoir participé à la construction du numéro 200, je parle en connaissance de cause. $C f$. Mathieu N., Clerc F. «La Sfer regarde sa revue : 200 numéros en 42 ans. Quel bilan? ». In Économie rurale, ${ }^{\circ} 200$, novembre-décembre 1990, p. 74-81.
}

relation de son travail non seulement avec l'actualité et l'action politique qui touchent son objet de recherche, mais aussi avec la posture théorique qu'il engage et qui le distingue d'autres écoles de pensée dans sa discipline ou dans les autres disciplines qui en traitent. Il doit en effet tout à la fois : 1. Lutter contre les stéréotypes qui parasitent l'interprétation des changements sociaux et la conception des politiques, et donc avoir un esprit critique très en éveil ;

2. Interroger inlassablement le réel avec des outils d'analyse toujours plus pertinents s'il veut avec rigueur suivre et énoncer les faits qui jalonnent cette évolution - dans ce cas celle de la ruralité dans sa dimension sociale et spatiale.

Il me faut aussi revenir sur les termes utilisés dans la demande, ceci non pas pour me légitimer mais pour dire en quoi et comment je me rattache aux catégories d'observateur, d'analyste et d'acteur du monde rural, et quelle est ma position par rapport à l'idée d'évènement et d'évolution. 


\section{Le monde rural Être observateur, analyste et acteur}

Observatrice...

Je veux l'être car l'observation est à la base de toute production de connaissances scientifiques, à condition, de mon point de vue, de définir cette observation à plusieurs échelles mises constamment en parallèle : le microcosme de la «commune », du « pays » ou du « quartier » qui permet l'approche holiste des rapports sociétés/espaces et sociétés/ natures; l'échelle méso de la région où apparaissent avec plus d'évidence les relations entre les villes et leurs campagnes ; l'échelle nationale où sont pensées les politiques agricoles et de développement rural ; l'Europe où se révèlent les différences de cultures politiques par rapport à la paysannerie (à la question agricole et alimentaire) et de définition de la ruralité comme de la conceptualisation des relations villes/ campagnes.

\section{Mais aussi analyste...}

Car passionnément soucieuse de l'interprétation de ces observations (statistiques et qualitatives comme dans l'Observatoire des rapports rural/urbain) ${ }^{2}$ et dans une attention constante à l'acte de concevoir, aux outils conceptuels de certaines disciplines - l'anthropologie et la géographie en particulier, à l'élaboration de concepts nouveaux... travail indispensable pour construire une juste interprétation de l'évolution de l'agriculture et du monde rural débarrassée des stéréotypes et des pesanteurs des interprétations idéologiques ou passées.

\section{Tout en étant acteur}

Mais suis-je ou ai-je été « acteur » de cette évolution? La réponse ne m'appartient pas. Tout au plus puis-je rappeler que malgré le système d'évaluation d'un chercheur au

2. Cf. Mathieu N., Robert M. Pourquoi un observatoire des rapports urbain/rural ? Observatoire des rapports entre rural et urbain, 4 pages, 1, décembre 1998, 6 p.
CNRS qui pousse au travail social clandestin, j'ai été «physiquement » impliquée avec d'autres ruralistes comme Françoise Bourquelot dans quelques luttes paysannes des années 1970 (dans la Nièvre avec les Paysans travailleurs ${ }^{3}$, dans les Landes avec les salariés agricoles du domaine de Solferino...). Etre actrice « activiste » dans le Mouvement de pays et le développement local des années 1980 aux côtés - entre autres - de Jacqueline Mengin et qui a mobilisé de nombreuses heures de travail dont il m'est difficile de juger l'efficacité par rapport au changement social lui-même. Prendre sa part dans l'action sociale - sans craindre l'immersion qui n'est pas contradictoire avec la distanciation - me semble jusqu'à aujourd'hui ${ }^{4}$ un moyen étonnant pour pénétrer les strates souvent cachées de l'évolution des sociétés et de leur rapport au monde. Finalement je revendique ma position d'acteur à travers mon métier de chercheur qui n'a jamais été dissocié de l'idée sartrienne qu'il existe un lien fort entre le scientifique et le politique et que l'action du chercheur est précisément de porter à la connaissance de tous les faits qui engagent des modifications des orientations et des trajectoires des acteurs individuels et collectifs. Autrement dit pour moi l'observation et l'analyse de toutes sortes de terrain y compris sa propre vie sont aussi des actes agissant, des actions.

Mais une fois ce pedigree esquissé - qui m'autorise à me constituer en « témoin » de « l'évolution de l'agriculture et du monde rural » français en particulier -, encore fautil que j'identifie ceux des évènements ou des évolutions observés que je considère comme suffisamment marquants pour infléchir les dynamiques rurales et agricoles. Il me faut repérer les faits qui sont porteurs de

3. Cf. Mathieu N. (1979). Coopération et affaire de cochons. Nouvelles campagnes, avril-mai, $\mathrm{n}^{\circ} 4$, p. 3-8.

4. Cf. La liste en 2008 «Vivre et agir à SaintDenis-Le-Gast » (550 habitants dans la Manche). 
changement et, en me référant aux concepts de continuité ou de rupture, celles des évolutions que je peux qualifier de «mutations ». Ceci implique d'ailleurs un certain rapport au temps et à l'histoire car il y aura sans nul doute plusieurs façons de rendre compte des évolutions de l'agriculture et du monde rural dans les « 59 ans de parution ininterrompue » d'Économie rurale.

\section{L'évolution du monde rural Savoir témoigner}

Dans ce retour critique de mon rapport à l'histoire de la ruralité que cette « commande » m'oblige à faire, il faut donc aussi que j'indique les fondements de ma posture théorique. C'est d'abord l'observation continue et dans la longue durée, la chronique des évènements de toutes sortes (catastrophes naturelles, crises et/ou créations d'activités économiques, évènements sociaux) et de tous niveaux (évènements familiaux, installation de nouveaux arrivants, initiatives individuelles et collectives mais aussi changements «globaux » politiques, économiques et environnementaux...) comme je l'ai préconisé dans l'Observatoire du Méjan. Mais c'est aussi le suivi à enveloppe spatiale constante des données statistiques disponibles comme par exemple celles de l'emploi en milieu rural, du rapport entre population agricole et population non agricole, voire de l'exclusion et de la pauvreté, etc. L'histoire qui m'intéresse est donc à la fois celle des évènements minuscules et du changement social comme fait total appréhendé au niveau d'une société micro locale et en même temps celle des tendances - on disait lourdes - qui parfois traduisent l'effet d'agrégation des comportements individuels mais qui suscitent aussi des phénomènes d'adaptation. Mais surtout, c'est dans l'articulation de ces deux types de temporalité que se dessine le futur, l'histoire étant pour moi un moyen de comprendre avant, d'anticiper, ce qui va changer. En somme une recherche concomitante des invariants - on dirait aujourd'hui plutôt des résiliences - et des discontinuités, ruptures et bifurcations

Alors que choisir dans cette longue observation du milieu rural dans son rapport à l'autre - l'urbain et la sphère politique européenne - comme dans ses rapports internes - les agriculteurs et les autres habitants des territoires ruraux ? Que choisir comme changement social rural marquant qui fasse le lien entre ces évènements minuscules observés par le petit bout de la lorgnette et ces faits si lourds qu'ils mobilisent tout à la fois le politique et l'opinion publique comme la mise en place des quotas laitiers, le découplage de la nouvelle PAC ou, plus que le premier choc pétrolier, la question du changement climatique en parallèle avec la raréfaction des ressources fossiles énergétiques?

\section{De l'événement minuscule au fait majeur pour imaginer l'avenir}

«L'évolution des modes d'habiter; un révélateur des mutations des sociétés rurales et urbaines ${ }^{5}$, c'est autour de ce titre que je choisis d'énoncer ce qui me semble être l'évolution majeure de l'économie et de la société rurale française : le changement dans le mode d'habiter les territoires ruraux. Cette réponse articule les deux tensions qui fondent mon intelligence du social : l'analyse continue d'évènements minuscules et quotidiens au fil du temps dans les territoires du quotidien que sont les localités rurales observées

5. Mathieu N. (2007). L'évolution des modes d'habiter : un révélateur des mutations des sociétés urbaines et rurales. Introduction. In Luginbühl Y. (Dir.), Nouvelles ruralités, nouvelles ruralités en Europe. Bruxelles, Bern, Berlin, Frankfurt am Main, New York, Oxford, Wien, Peter Lang, p. 25-45. 
depuis plus de trente ans ${ }^{6}$ et qui renvoient pourtant aux évolutions globales comme à la crise de la vache folle ou au réchauffement climatique; la lente construction d'un concept - le mode d'habiter - me permettant de penser ensemble les rapports entre les lieux (les habitats de l'homme) avec leur matérialité physique, leurs valeurs sensibles d'une part, et les individus qui les habitent, les praticiens des lieux d'autre part. C'est en effet en interrogeant ce qu'habiter veut dire, et du point de vue des lieux et milieux, et du point de vue des individus qui les habitent, c'est en observant toutes les formes par lesquelles les lieux sont habités - circuler, travailler, se loger et se nourrir, vivre ensemble - que s'est imposée à moi, peu à peu, une interprétation dominante de l'évolution des sociétés locales rurales : l'éloignement toujours plus grand des agriculteurs de leur conscience d'être des praticiens des lieux, et d'avoir à reconstruire un lien social local avec d'autres qu'eux.

Ce qui frappe en effet le plus l'observatrice, l'analyste et l'acteur du « rural » que je suis devenue au fil des ans c'est cette dissociation lente, progressive et presque inéluctable qui s'est produite au niveau local dans les innombrables communes qui forment le tissu de la ruralité française, entre les agriculteurs de métier et les autres habitants des territoires ruraux.

Il me revient le souvenir de Bernard Kayser dans une des conférences qu'il prononça au cours des journées de l'Association des ruralistes français (ARF) dans les années 1980 : il voyait dans la capacité de nouer des alliances locales de

\footnotetext{
6. Il s'agit essentiellement de l'observation continue de l'articulation entre le local et le global, entre les individus dans tous leurs lieux de vie dans trois terrains mis en parallèle : la société méjanaise (Causse Méjan en Lozère), le canton de Gavray et la commune de Saint-Denis-le-Gast dans la Manche et en contrepoint des microsites parisiens et rouennais.
}

proximité, voire de voisinage entre agriculteurs et non-agriculteurs le principe même de la nouvelle ruralité, de la « renaissance rurale ». Il me semble que l'occurrence de cette nouvelle alliance à établir dans les lieux de vie ruraux, là où la cohabitation et le partage des ressources est à la fois inévitable et porteur de contradictions et de conflits ne s'est pas produite - sauf dans des lieux exemplaires invariablement cités en exemple- et aura de plus en plus de mal à s'établir depuis le monde agricole alors que par le biais de la question de l'aliment sain et savoureux, ruraux non-agricoles et citadins sont de plus en plus demandeurs mais aussi acteurs (initiateurs) du changement.

Du côté des habitants agriculteurs comme du côté des autres habitants d'une localité ce sont des faits minuscules, la recherche du sens de moments de détailstemps qui m'ont conduite à considérer ce détachement, cette dissociation des rapports sociaux locaux comme l'évolution la plus marquante des nouvelles ruralités.

Confrontés aux injonctions contradictoires de la mondialisation et des directives européennes la conscience ou la capacité des agriculteurs d'habiter - au sens donné plus haut - un territoire se réduit. L'agriculteur formaté par ces injonctions est de moins en moins dans un rapport amoureux et ménager avec le milieu naturel et la matérialité sur lesquels sa spécificité est fondée. Il suffit de rapprocher les premières pages du Contrat naturel de Michel Serres et sa description de la paysannerie de ce que l'on observe du comportement physique des agriculteurs dans leurs terres ou de leur consommation. Du haut de leurs tracteurs puissants et sophistiqués, ils ne semblent plus toucher le sol et, pressés de produire, ils n'ont cure d'entretenir les haies et les talus, de contrôler l'embroussaillement s'ils n'ont pas les «compensations » correspondant à ce travail devenu inutile. Faute de temps pour faire le jardin ou pour 
penser l'auto-approvisionnement en termes domestiques et pas seulement animal, ils sont les premiers consommateurs des grandes surfaces pour le lait UHT, voire les carottes râpées vendues au rayon produits préparés. Ce qu'ils produisent est mentalement et réellement dématérialisé : leurs relations avec leur fonction nourricière comme avec les milieux naturels et vivants qui les supportent se distendent.

Inversement, il m'apparaît que le désir « insoutenable » de nature dont on ne reconnaît l'existence que pour les « urbains » comme si habiter la ville excluait tout rapport local à la nature se retrouve aussi chez des ruraux « de souche » partageant avec les nouveaux arrivants urbains - mais aussi souvent d'origine rurale - le goût des randonnées sur les chemins communaux, des nourritures « locales » dont on connaît la qualité et des produits du jardin.

À l'opposé des pratiques des agriculteurs, ce que l'on pourrait appeler le nouveau petit peuple des campagnes et des bourgs cultive pour se nourrir des produits et des semences retrouvant de façon spontanée des pratiques de vente ou d'échange ce qui finit par constituer une économie informelle de type paysan à côté du groupe social des agriculteurs.

D'ailleurs, parallèlement à sa technicisation et à une dépendance de plus en plus grande vis-à-vis des subventions européennes, le sentiment que la dignité du métier d'agriculteur pour soi-même et pour les autres devient de plus en plus difficile à garder. Certes les efforts fait pour rétablir cette image autour de personnages comme A. Pochon et l'agriculture durable ou par des opérations de promotion des liens ville/campagne comme cela s'est produit dans le Vexin français tentaient de réhabiliter la fonction des agriculteurs en insistant sur la fonction nourricière retrouvée ou sur le gardiennage de la nature et du paysage! Mais ces évènements sont restés éphémères ou plutôt sont restés dans le cadre global de l'image et du pouvoir des agriculteurs et dans une sphère a-locale des relations entre les agriculteurs et les autres : observé au niveau local le métier d'agriculteur reste perçu comme un métier qui ne doit pas sa force à ses propres forces mais au système de la PAC et de ses réformes en relation avec l'OMC.

\section{Pour conclure}

Cela ne veut pas dire que les autres habitants ruraux -malgré leurs efforts souvent au sein d'associations- parviennent à reconstruire ce lien social local nécessaire pour vivre ensemble et organiser un nouvel usage social des lieux. On observe au contraire chez les non-agriculteurs des phénomènes de repli sur soi, le « cultiver son jardin », « bien vivre et travailler dans sa maison avec les $\mathrm{NTIC}^{7}$ » et « passer inaperçu quand on est pauvre » devenant la nouvelle forme de l'individualisme rural. Mais la question critique n'est-elle pas comment agriculteurs et habitants résidents peuvent-ils retrouver le sens du « commun » : partager les ressources naturelles, surmonter les divergences culturelles et d'intérêt entre les nouveaux ruraux non originaires et sans liens familiaux et les locaux « légitimes », lutter ensemble contre la montée de la pauvreté et de l'exclusion en milieu rural ? Comment construire une nouvelle cohabitation du milieu rural, une ruralité durable ? Je ne peux m'empêcher de penser que, en retrouvant l'esprit des lieux et le respect de leurs nouveaux voisins, les agriculteurs seront une fois de plus moteurs de cette mutation du mode d'habiter rural. Du travail d'observation et d'analyse pour les futurs numéros d'Économie rurale.

7. Nouvelles technologies de l'information et de la communication. 